\title{
La introducción de la vacuna contra la hepatitis A en el Calendario Nacional de Vacunación: una nueva realidad Hepatitis A immunisation in the argentinean mandatory schedule
}

\author{
Dra. Ángela Gentile ${ }^{a}$ Dra. Margarita D. Ramonet ${ }^{b}$ y Dra. Mirta Ciocca ${ }^{c}$
}

\begin{abstract}
RESUMEN
La hepatitis A (HA) presenta una evolución benigna, pero en ocasiones puede causar una insuficiencia hepática aguda con necesidad de trasplante. En 2003, en un consenso realizado en la Sociedad Argentina de Pediatría, se recomendó la incorporación de la vacuna contra la HA en el calendario nacional. Esto se concretó por decreto ministerial y se efectivizó a partir del 1 de junio de 2005, con la obligación de aplicar una dosis de la vacuna a todos los niños al año de edad. Desde entonces, se registró una disminución marcada de casos y no se documentaron cuadros de insuficiencia hepática aguda desde el año 2006. Los estudios de seguimiento mostraron, hasta el momento, una circulación viral baja y la persistencia de anticuerpos hasta los 5 años posteriores a su introducción.

Palabras clave: hepatitis A, inmunización, insuficiencia hepática aguda.
\end{abstract}

\section{SUMMARY}

Hepatitis A (HA) presents a benign evolution, but occasionally some patients develop a more severe disease. Previously to 2005 hepatitis A was an important cause of acute liver failure (ALF) and hepatic transplant. In 2003, a consensus in the Argentinian Pediatrics Society was done; it had just recommended the inclusion of the vaccine in the mandatory immunisation schedule. This was issued by the Health Ministery, and was applied on June $1^{\text {st }}, 2005$. The schedule was one dose at the age of one year of age. Since then, an important reduction of HA was registered, without any case of ALF since 2006. Follow-up studies so far showed low viral circulation and persistence of antibodies to 5 years later.

Keywords: hepatitis A, immunization, acute liver failure.

http:/ /dx.doi.org/10.5546/aap.2013.155

\section{INTRODUCCIÓN}

La infección por el virus de la hepatitis A (VHA) se considera mundialmente un problema sanitario importante debido a su morbimortalidad. ${ }^{1,2}$ Es una de las causas más frecuentes de hepatitis viral aguda en gran parte del mundo. Su incidencia está relacionada con los niveles de hi- giene y saneamiento ambiental, el desarrollo socioeconómico y el nivel de educación de la población. 3,4

\section{Epidemiología}

Se trata de una enfermedad inmunoprevenible, transmitida por un virus simple, no encapsulado, de la familia Picornaviridae, género $\mathrm{He}$ patovirus. ${ }^{4}$ Todas las cepas del virus identificadas hasta la fecha son inmunológicamente indistinguibles y pertenecen a un solo serotipo. En nuestro país, el subgenotipo 1A sigue siendo el único hallado, tanto en las formas habituales como en las fulminantes, según los estudios realizados en el Instituto Malbrán. ${ }^{5}$ Dada la actual epidemiología de la enfermedad en la Argentina, con tasas que han decrecido sustancialmente, la identificación de los genotipos permitirá el seguimiento de los casos en la ocurrencia de brotes a partir de casos importados. ${ }^{6}$ La vía de transmisión es oral-fecal a partir de la ingestión de partículas virales infectantes por contaminación de las manos, los alimentos o el agua con heces provenientes de un individuo infectado; no evoluciona a la cronicidad. ${ }^{7}$ La infección se mantiene en la naturaleza debido a su transmisión, a partir de individuos infectados agudamente, a huéspedes susceptibles; en este sentido, es muy importante el papel que desempeñan los niños menores de 6 años, y especialmente los menores de 2 años, en la difusión del virus al desarrollar formas asintomáticas (70\%). ${ }^{7-9}$ Ellos son el real reservorio de la enfermedad.

De acuerdo con la Organización Mundial de la Salud (OMS), hubo un incremento de casos desde el año 1990, 
en que se notificaron 117 millones de casos, en el año 2005, con 121 millones, fundamentalmente en dos grupos de edad: de 2 a 14 años y mayores de 30 años. Cabe hacer notar que se produce un paradójico incremento en la incidencia y la gravedad de la enfermedad cuando las condiciones socioeconómicas y sanitarias mejoran; debido a este cambio, el contacto con el virus de la hepatitis A se da en edades mayores, en las cuales la enfermedad es esencialmente sintomática, ictérica, con lo cual la notificación de casos aumenta. Sin embargo, la fuente de infección del VHA se presenta como indefinida en más del $50 \%$ de los casos. ${ }^{4}$

Es importante destacar que si bien es una enfermedad de transmisión oral-fecal, no se puede controlar solo con medidas de saneamiento ambiental; es fundamental complementarlas con el uso universal de la vacuna.

\section{Vacunas}

La exitosa propagación del VHA en el cultivo de tejidos, en el año 1979, permitió el desarrollo de varias vacunas de virus inactivados. Desde 1995, la hepatitis A es una enfermedad prevenible con la vacunación; la estrategia de inmunización universal es costo-eficaz. El trabajo de Ellis y cols., que analizó los datos del país, estimaba que la política de vacunación con una dosis reduciría la probabilidad de cada miembro de la cohorte de padecer hepatitis A sintomática, en 50 años, de $7,2 \%$ a $4,1 \%$. Un esquema con una dosis única logra los estándares aceptados de eficacia en función del costo en todas las regiones del país y reduciría los costos en las regiones nordeste, central y sur. ${ }^{4,9-17}$

En general, la incorporación de esta vacuna en los calendarios nacionales está recomendada en países con una tasa de incidencia mayor de 20 casos/100 000 habitantes, sugerida en países con tasas de 10-19/100 000 y no recomendada cuando la incidencia es menor de 10 casos $/ 100000 .{ }^{18}$

Bell y cols. dicen: "Como la HA no produce una infección crónica y los seres humanos son los únicos reservorios naturales del virus, es probable que la reducción en la incidencia pueda ser sustentada por el mantenimiento de altos niveles de inmunidad de la población mediante la vacunación universal en la niñez, ya que es poco probable que se obtenga con la aplicación de una vacunación selectiva a grupos de riesgo, o programas a corto plazo como los que se realizan para el control de brotes epidémicos" ${ }^{18}$ Los beneficios de la vacunación universal en la infancia se dan especialmente cuando se realiza antes de la etapa de riesgo, interrumpiéndose así la transmisión del virus entre los niños en edad escolar temprana, que son los reales reservorios de la enfermedad. Así, un niño inmunizado no solo está protegido él

Figura 1. Casos y tasas de la hepatitis A, período 2000-2011, Ministerio de Salud, Argentina

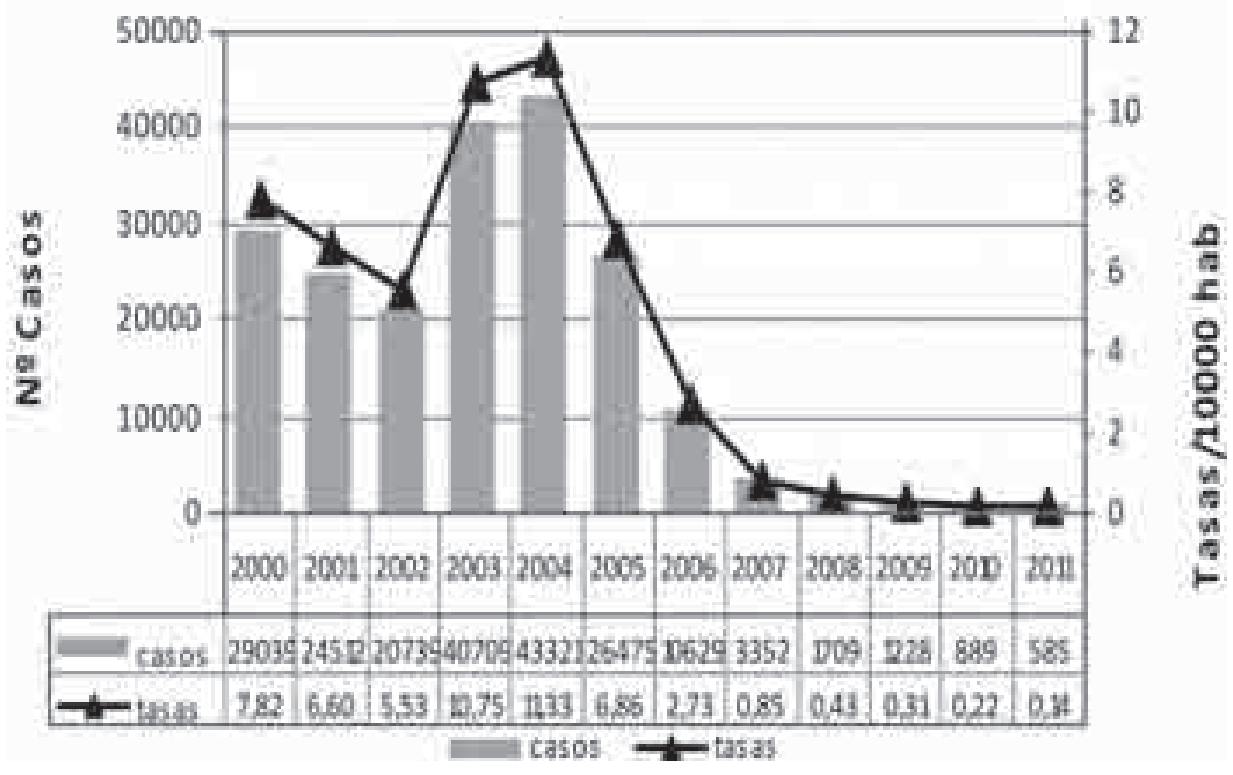


mismo de la enfermedad, sino que también protege a otros niños de diferentes edades y adultos susceptibles, logrando de ese modo eliminar la fuente de infección. Es una vacuna que confiere inmunidad de rebaño. ${ }^{13}$

El primer país del mundo que utilizó la inmunización universal fue Israel, cuya tasa anual de incidencia era del 50,4/100 000 durante los años 1993-1998. Si bien es un país más pequeño que la Argentina, tiene dos poblaciones de muy diferente etnia, judíos y beduinos, cuyas condiciones socioeconómicas también son diferentes. En julio de 1999, comenzó el programa de inmunización universal con dos dosis de vacuna HA, a los $18 \mathrm{y}$ 24 meses, en forma gratuita. La incidencia anual durante 2002-2004 fue del 2,5/100 000, lo que representa más del 95\% de reducción por cada año con respecto al período 1993-1998. Es interesante destacar que esta importante declinación se observó no solo en los niños de 1 a 4 años $(98,2 \%)$, sino también en todas las edades: 5 a 9 años: $96,5 \%$; 10 a 14 años: 95,2\%; 15 a 44 años: 91,3\%; 45 a 64 años: 90,6\% y mayores de 65 años: $77,3 \%$, lo cual demuestra claramente la protección de rebaño conferida por la vacunación. ${ }^{19}$

\section{Antecedentes en la Argentina}

Antes de 2005 la Argentina era un país de endemicidad intermedia, con áreas de alta endemicidad y desarrollo de brotes epidémicos, como el acontecido en los años 2003 y 2004. Las tasas de infección eran de 173,7/100 000 habitantes, la edad de mayor prevalencia estaba entre los 5 y los 9 años, y la HA era una causa importante de insuficiencia hepática aguda y necesidad de trasplante hepático (Figuras 1 y 2). ${ }^{7,8,20}$

En un estudio seroepidemiológico realizado en distintas regiones del país en 1997, en una población de 3699 niños de 1 a 10 años, sin antecedentes de hepatitis aguda, la prevalencia de anticuerpos IgG-VHA fue del 51,5\%, con diferencias marcadas entre Tucumán, con una prevalencia del $81,4 \%$, y la Ciudad Autónoma de Buenos Aires, con el $29,4 \%$, mientras que en Trelew fue del $41,9 \%$, en San Justo (Provincia de Buenos Aires), del 57,8\% y en Rosario, del 46,5\%, lo que demuestra su relación con las diferentes condiciones socioeconómicas y ambientales. ${ }^{21}$

Para conocer la situación de la infección por el VHA en la Argentina durante el período prevacunación, cabe destacar la experiencia del Hospital Nacional Profesor Alejandro Posadas, sobre el diagnóstico y seguimiento de 4226 pacientes con hepatitis aguda durante un período de 18 años. La edad promedio de esa población fue de 7,3 años (1 a 18 años), siendo el 77\% menor de 10 años. La etiología más frecuente fue la HA, en el 92\% de los casos, mientras que el $6 \%$ fue indeterminada y solo el $2 \%$ fue causada por el virus de la hepatitis B (VHB). ${ }^{22}$

En relación con la insuficiencia hepática agu$\mathrm{da}$, es interesante mencionar el estudio realizado

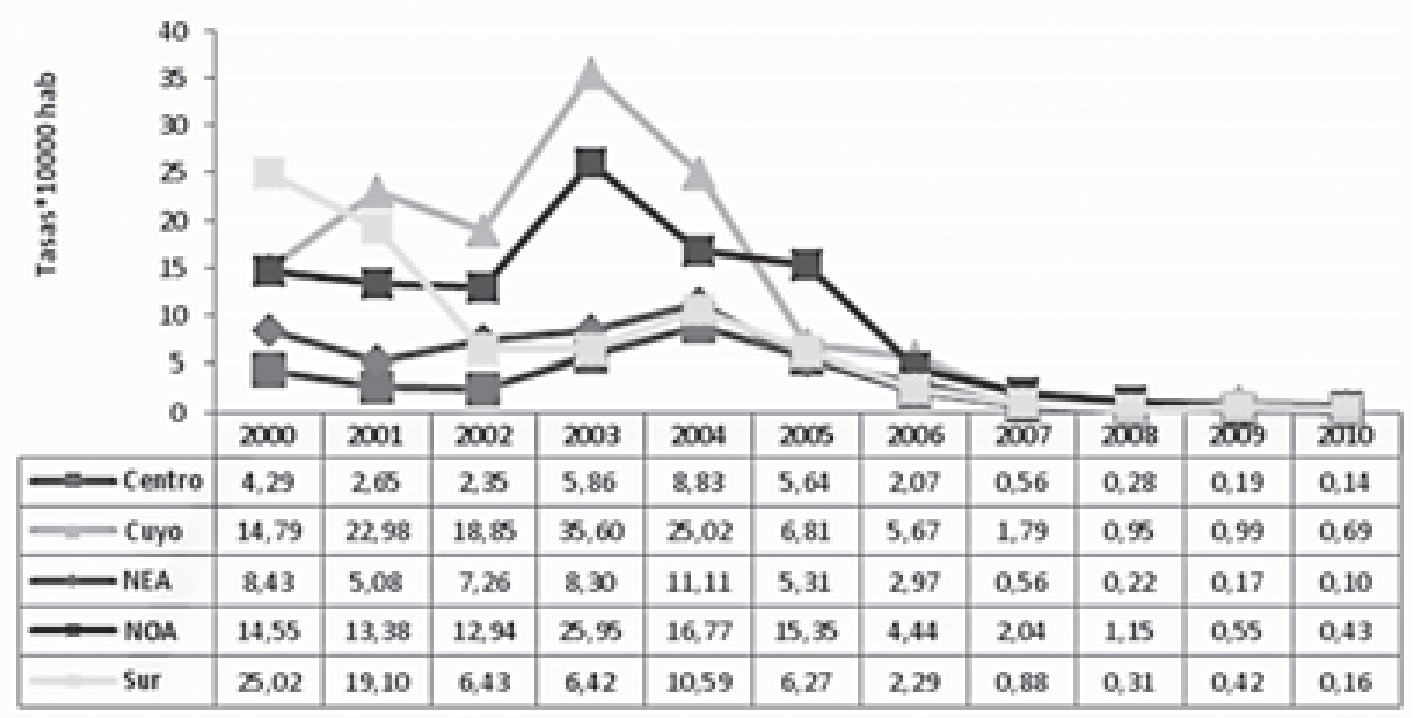


por los hospitales Garrahan y Posadas en 210 pacientes atendidos por esa causa en dichos centros en un período de 20 años. La distribución etiológica fue la siguiente: VHA: $61 \%$, indeterminada: $32 \%$, y $7 \%$ obedecieron a varias causas, como VHB, tóxica, hepatitis autoinmunitaria, etc. La media de edad fue de 5,3 años, con un rango de 1 a 17,4 años; 87\% fueron menores de 10 años y $63,5 \%$, menores de 5 años. De estos 210 pacientes pediátricos 59 sobrevivieron ( $68 \%$ por VHA), 61 fallecieron ( $54 \%$ por VHA) y 90 requirieron un trasplante hepático (61\% por VHA) ${ }^{23}$

La HA en nuestro país era entonces la primera causa de hepatitis fulminante y de trasplante hepático en la población pediátrica.

\section{Implementación de la vacunación en el país}

Debido a este escenario epidemiológico, los Comités Nacionales de Infectología y Hepatología elaboraron un Consenso en el marco de la Sociedad Argentina de Pediatría (SAP), en diciembre de 2003, donde fueron convocados los diferentes comités, grupos de trabajo y subcomisiones relacionados con la temática. En dicho Consenso se recomendó la incorporación de la vacuna HA en el calendario nacional con un esquema de dos dosis, ${ }^{20,24}$ teniendo en cuenta algunos puntos fundamentales:
- Prioridad de la enfermedad y su control.

- Características de la vacuna.

- Factibilidad programática.

- Suministro de la vacuna.

Se analizaron, entonces, tres posibles esquemas:

1. Dos dosis a partir del año de vida con un intervalo de seis meses entre ellas (12 y 18 meses).

2. Una dosis única al año de edad.

3. Dos dosis, a los 12 meses y al ingreso escolar respectivamente.

Ya en ese momento el Programa Nacional de Inmunizaciones había incorporado la utilización operativa de la vacuna HA para el manejo de brotes; la primera experiencia programática fue durante las inundaciones en la Provincia de Santa Fe.

La inclusión de la vacuna HA en el calendario nacional de inmunizaciones (Resolución Ministerial 653) se realizó el 20 de abril de 2005 y se efectivizó a partir del 1 de junio del mismo año, para todos los niños nacidos en 2004, es decir, que cumplieran el primer año de vida a partir del 1 de enero de 2005..$^{25}$

El esquema de vacunación propuesto en ese momento, de una dosis de vacuna HA al año de edad, fue sustentado en las siguientes premisas:

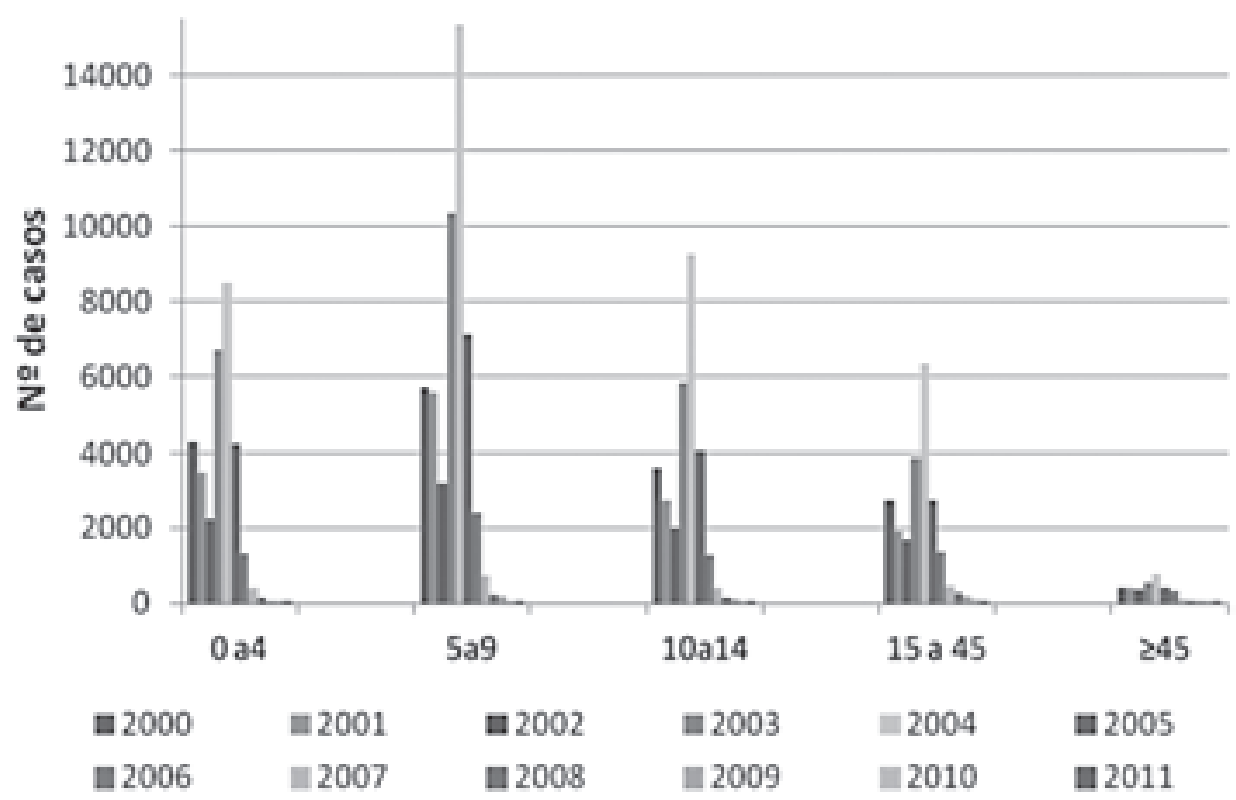


- Una sola dosis genera una respuesta inmuntaria protectora en el 95\% al 99\% de los vacunados a los 30 días (Gentile A. Comunicación personal).

- La amplia experiencia con el uso de una dosis de vacuna para controlar brotes epidémicos.

- La amplia circulación del VHA en nuestro medio favorece la exposición al virus y actúa como un "refuerzo" natural en los vacunados.

Los niños menores de un año están protegidos por los anticuerpos maternos. A partir del año de edad, la infección produce un gran número de formas asintomáticas, constituye el reservorio del virus y favorece la transmisión a otros grupos susceptibles.

Desde el punto de vista operativo, se consideró la concurrencia de los niños a los 12 meses de edad para la aplicación de la vacuna triple viral, en cumplimiento con el calendario nacional de vacunación. Al no haber contraindicaciones para el uso simultáneo de ambas vacunas, se propuso administrarla en esa oportunidad y simplificar así la logística para su aplicación.

El documento destaca las siguientes acciones para implementar:

- Fortalecer la vigilancia epidemiológica de la HA.

- Evaluar el impacto del programa a través de un protocolo de seguimiento y, de acuerdo con sus resultados, se podría llegar a incluir un refuerzo o bien continuar con el esquema actual.
- Mantener de forma continua la difusión de las medidas de higiene personal, como también la potabilización del agua y el tratamiento adecuado de las excretas.

- Desarrollar la coordinación y cooperación intersectorial e interjurisdiccional para el mejoramiento del saneamiento básico.

\section{Etapa posvacunación}

Tras la incorporación de la vacuna en el calendario nacional de inmunizaciones, se registró una declinación marcada de las tasas que disminuyeron de 113,3/100000 en el año 2004 al $1,4 / 100000$ en $2011 .{ }^{26}$ Durante todo este período la cobertura de vacunación fue mayor del $90 \%$ en todo el país. ${ }^{27}$

Si se analizan los porcentajes de notificación de hepatitis A según grupos etarios (Figuras 3 y 4) se observa un claro desplazamiento de los casos confirmados hacia los grupos de mayor edad a partir de la introducción de la vacuna; así, en el año 2000 la mayor incidencia correspondía al grupo de 5 a 9 años, mientras que en 2010 pasó a ser el de 15 a 44 años. Esta situación epidemiológica obliga a realizar una estrecha vigilancia epidemiológica y estudios serológicos a fin de dar seguimiento a la estrategia adoptada por el país; tal el compromiso enunciado por el Ministerio de Salud en 2005. Es esperable que aparezcan casos en grupos no vacunados, pero la incidencia en tales grupos debe ser baja ya que, por un lado, la

FIGURA 4. Proporción de notificaciones de hepatitis A por grupo etario y por año, período 2000-2011, Ministerio de Salud, Argentina

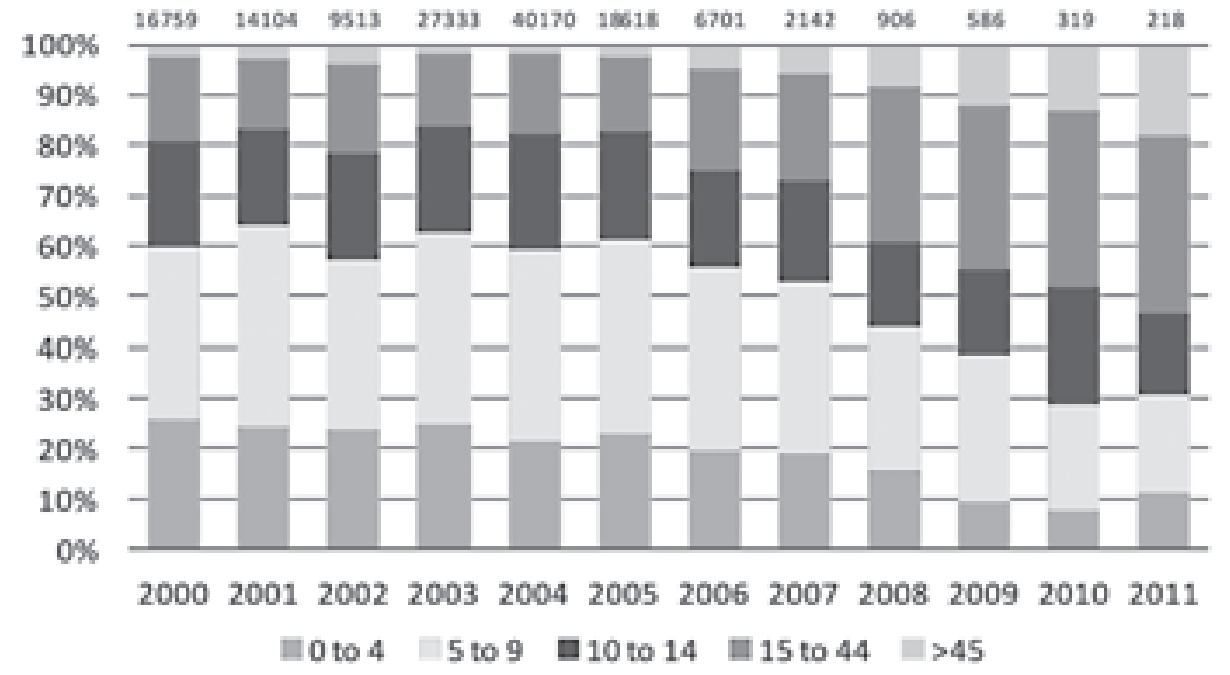


vacuna confiere inmunidad de rebaño y, por otro lado, cada vez más el país tiene su población vacunada. De hecho, en la actualidad todos los menores de 9 años deberían estarlo.

Recientemente, en una revisión retrospectiva de cuatro centros pediátricos de Buenos Aires, se analizó la frecuencia y evolución de la insuficiencia hepática aguda asociada al VHA, así como los casos de trasplante hepático, antes y después de la implementación de la vacunación universal en el país. Durante el período prevacunación (marzo de 1993 a julio de 2005), 54,6\% de los casos de insuficiencia hepática aguda fueron asociados al VHA, mientras que en el período posvacunación (agosto de 2005 a octubre de 2006), solo lo fue el $27,7 \%$, sin haberse notificado casos desde noviembre de 2006 hasta diciembre de $2008 .^{28}$ Esta situación permaneció sin cambios hasta la actualidad.

Es interesante mencionar que solo 11 países $(6 \%)$ tienen incorporado en el calendario nacional de inmunizaciones la vacuna HA con la estrategia de dos dosis y la Argentina es, por el momento, el único con un esquema de monodosis.

\section{Protocolos de seguimiento}

Luego de un trabajo intensivo y conjunto con el Programa Nacional de Inmunizaciones del Ministerio de Salud de la Nación, en el año 2011 se concretaron dos estudios multicéntricos; en el Laboratorio Nacional de Referencia INEI-ANLIS Dr. Carlos Malbrán se realizaron las determinaciones serológicas.

El primero de ellos evaluó la circulación viral, sustentado conceptualmente en que si ha habido una disminución en nuestro medio de la circulación del VHA, menos mujeres habrán tenido contacto con el virus durante el embarazo; en consecuencia, pasarán un menor tenor de anticuerpos a su feto y el recién nacido entonces presentará una menor concentración de IgG anti-VHA.

Según los datos presentados se halló una circulación viral baja, ya que el $69,5 \%$ de los niños estudiados tenían niveles de IgG anti-VHA menores de $10 \mathrm{mUI} / \mathrm{mL}$, es decir, que el 29,5\% (IC $95 \% 25,2$ a 33,8 ) de los niños tuvieron títulos de anticuerpos seroprotectores (IgG anti-VHA $>10$ $\mathrm{mUI} / \mathrm{mL}$ ). La determinación de IgM anti-VHA fue negativa en todos los casos. Este hallazgo es fundamental, pues permite descartar una infección reciente y concluir que los anticuerpos detectados son por transferencia materna. ${ }^{29}$

El segundo protocolo investigó la persistencia de los anticuerpos luego de 4 años de la vacunación en niños de entre 4 y 5 años que habían reci- bido una sola dosis al año de vida; $93 \%$ de ellos presentaron títulos protectores $(>10 \mathrm{mUI} / \mathrm{mL})$.

La inmunidad conferida por la vacuna es no solo humoral sino también celular, lo que aseguraría una protección a largo plazo. El nivel de anticuerpos actúa como un correlato de protección, por lo que podemos asegurar que estas cohortes después de 4 años están bien protegidas. ${ }^{30}$

Estos datos fueron presentados por el Ministro de Salud de la Nación el 12 de abril de 2012 en Ginebra y la estrategia fue respaldada por el organismo internacional. ${ }^{31}$ El Grupo de Expertos en Asesoramiento Estratégico sobre Inmunización de la OMS (SAGE) publicó un documento de posición en el cual recomienda la integración de esta vacuna a los calendarios nacionales de vacunación para los niños a partir del año de edad con una dosis única teniendo en cuenta la incidencia de la enfermedad y los análisis de costo-eficacia.

Por el momento, con estas evidencias podemos concluir que no sería necesaria la aplicación de una segunda dosis en el calendario nacional. Sin embargo, esta estrategia debe ser seguida en el tiempo con estudios serológicos bianuales y con una exhaustiva vigilancia epidemiológica de la enfermedad.

\section{BIBLIOGRAFÍA}

1. Yazigi NA, Balistreri W. Acute and chronic viral hepatitis. En: Suchy FJ, Sokol RJ, Balistreri WF (eds). Liver disease in children. $2^{\text {nd }}$ Ed. Lippincott: Williams and Wilkins; 2001. Págs.365-428.

2. Koff RS. Clinical manifestations and diagnosis of hepatitis A virus infection. Vaccine 1992;(suppl 1):S15-S17.

3. Ciocca M. Clinical course and consequences of hepatitis A infection. Vaccine 2000;18:S71-S74.

4. WHO immunological basis for immunization series. Module 18: Hepatitis A. Genova: WHO; 2011.

5. Munné MS, Vladimirsky S, Otegui L, Soto S, et al. Molecular characterization of Hepatitis A virus isolates from Argentina. J Med Virol 2007;79:887-94.

6. American Academy of Pediatrics; Hepatitis A. En: Pickering LK, ed. Red Book: 2009. Report of the Committee on Infectious Diseases. 28 ${ }^{\text {th }}$ ed. Elk Grove Village, Il: American Academy of Pediatrics; 2009. Págs. 329-37.

7. Ramonet M. Hepatitis A. Libro Azul de Infectología Pediátrica. $3^{\mathrm{a}}$ Ed. Buenos Aires: Sociedad Argentina de Pediatría; 2007. Págs. 435-48.

8. Ramonet M, Ciocca M. Situación de la infección por el virus de la hepatitis A en Argentina: antes y después de la implementación de la vacuna. GED 2007;26:207-12.

9. Debray D, Cullufi P, Devictor D, Fabre M, et al. Liver failure in children with Hepatitis A. Hepatology 1997;26:1018-22.

10. Jacobs RJ, Greenberg DP, Koff RS, Saab S, et al. Regional variation in the cost-effectiveness of childhood hepatitis A. Immunization Pediatr Infect Dis J 2003;22(10):904-14.

11. Rosenthal P. Cost-effectiveness of hepatitis A vaccination in children, adolescents, and adults. Hepatology 2003;37:44-51.

12. André F, Van Damme P, Safary A, Banatvala J. Inactivated 
hepatitis A vaccine: immunogenicity, efficacy, safety and review of official recommendations for use. Expert Rev Vaccines 2002;1(1):9-23.

13. Averhoff F, Shapiro CN, Bell BP, Hyams I, et al. Control of hepatitis A through routine vaccination of children. JAMA 2001;286(23):2968-73.

14. Innis $\mathrm{B}$, Smithham $\mathrm{R}$, Kumasol $\mathrm{P}$, Laorakpongse $\mathrm{T}$, et al. Protection against hepatitis A by inactivated vaccine. JAMA 1994;271:1328-34.

15. Centers for Disease Control and Prevention. Prevention of Hepatitis A through active or passive immunization: recommendations of the Advisory Committee on Immunization Practice (ACIP). MMWR. Morb Mortal Wkly Rep 1999;48(RR-12):1-37.

16. Ellis A, Rüttimann R, Jacobs R, Meyehoff A, et al. Costeffectiveness of childhood hepatitis A vaccination in Argentina: a second dose is warranted. Pan Am J Public Health 2007;21(6):345-55.

17. Ginsberg GM, Slater PE, Shouval D. Cost-benefit analysis of a nationwide infant immunization programme against hepatitis A in an area of intermediate endemicity.J Hepatol 2001;34:92-99.

18. Bell BP, Shapiro CN, Alter MJ, Moyer LA, et al. The diverse patterns of hepatitis A epidemiology in the United States: implications for vaccinations strategies.J Infect Dis 1998;178:1579-84.

19. Dagan R, Leventhal A, Anis E, Slater P, et al. Incidence of hepatitis A in Israel following universal immunization of toddlers. JAMA 2005;294(2):202-10.

20. Gentile A, Ramonet M, Ellis A. Análisis sobre la necesidad de incorporación de la vacuna contra la hepatitis A en la Argentina. Consenso Organizado por la Sociedad Argentina de Pediatría. Arch Argent Pediatr 2004;102(6):497-8.

21. González J, Fay O, Cañero-Velasco C, Fernández E, et al. Infección por el virus de la Hepatitis A en niños en Argentina. Ensayo piloto. Acta Gastroenterol Latinoam 1997;27:331-4.
22. Ramonet M, Gómez S, Morise S, Caglio P, et al. Acute hepatitis A Virus. Association with fulminant hepatic failure and autoimmune hepatitis in pediatric population. J Pediatr Gastroenterol Nutr 2000;31:S116.

23. Ciocca M, Ramonet M, Cuarterolo M, López S, et al. Prognostic factors in pediatric acute liver failure. Arch Dis Child 2008;93:48-51.

24. Gentile A. Theneed for an evidence-based decision-making process with regard to control of hepatitis A.J Viral Hepat 2008;15(Suppl 2):16-21.

25. Argentina. Ministerio de Salud y Ambiente. Resolución 653/05. Boletín Oficial de la República Argentina 2005; 113 (30677).

26. Ministerio de Salud y Ambiente. Hepatitis A. Boletín Epidemiológico Periódico. Edición Especial. Abril 2005; 3.

27. Argentina. Ministerio de Salud de la Nación. Secretaría de Promoción y Programas Sanitarios. Boletín Integrado de Vigilancia. Noviembre, 2011.

28. Cervio G, Trentadue J, D' Agostino D, Luque C, et al. Decline in $\mathrm{HAV}$-associated fulminant hepatic failure and liver transplant in children in Argentina after the introduction of a universal hepatitis A vaccination program. Hepatic Medicine: Ev Res 2011;3:99-106.

29. Vizzotti C, Gentile A, González J, Ramonet M, et al. Hepatitis A virus circulation after universal single dose vaccination in argentinean children. $14^{\text {th }}$ International Symposium on Viral Hepatitis and Liver Disease (ISVHLD) Shanghai, China; 24 de junio 2012.

30. Vizzotti C, Gentile A, González J, Rearte A, et al. High persistence of protective antibodies againts hepatitis after universal single dose vaccination in Argentina. $14^{\text {th }}$ International Symposium on Viral Hepatitis and Liver Disease (ISVHLD) Shanghai, China, 24 de Junio 2012.

31. WHO. Updated position on hepatitis A vaccination. [Acceso: 23 enero 2013]. Disponible en: http:/ / www.who.int/ csr/disease/hepatitis/publications/en/index.html. 